\title{
The Status of Family Resilience: Effects of Sustainable Livelihoods in Rural China
}

\author{
Bo Yang ${ }^{1} \cdot$ Marcus W. Feldman ${ }^{2}$ (D) Shuzhuo $\mathrm{Li}^{3}$
}

Accepted: 10 October 2020 / Published online: 26 October 2020

(c) The Author(s) 2020

\begin{abstract}
Family resilience is the capacity of a family to mitigate adversity using its resources, structure, and internal connections. In rural China, where sustainable livelihoods are changing rapidly because of urbanization and migration, it is not clear how family resilience interacts with sustainability of livelihoods. This study of rural Chinese families classifies individual's perceived family resilience and discusses how sustainable livelihoods influence this classification. A multilevel survey of rural families reveals three categories of perceived resilience in families: perceived optimistic families, perceived cooperative families, and perceived pessimistic families. Sustainable livelihoods, including natural, social, financial, and human capitals, have significant impacts on this classification of perceived family resilience. We conclude that rural families in China vary in their levels of family resilience, and the more physical and financial capital they have, the more internal cooperation there is within families. The more natural and traditional social capital families have, the less internal cooperation there is within families. The more human resources families have, the more resilience they have.
\end{abstract}

Keywords Family resilience $\cdot$ Rural families $\cdot$ Sustainable livelihood $\cdot$ China

\section{Introduction}

Family resilience is the capacity of a family to survive under adversity as a function of family resources, family structure, and within-family connections (Walsh 2003). Families that experience the risk of natural disasters use resilience to acquire necessary resources, adapt to changes, and therefore operate sustainably (Gallopín et al. 2006). Family resilience also includes the family's ability to deal with social challenges. In China, families are confronted with rapid social and economic change during which they need more resilience to acquire resources and survive. The first change is that the Chinese traditional family, where

Marcus W. Feldman

mfeldman@stanford.edu

1 International Business School, Shaanxi Normal University, Xi' an 710119, China

2 Department of Biology, Stanford University, Stanford, CA 94305, USA

3 Institute for Population and Development, Xi' an Jiaotong University, Xi' an 710049, China 
more than two generations live together, has become smaller after 40 years of the national family planning strategy. In 2017, $67.6 \%$ of families had only three or fewer members, indicating that China's traditional pattern of large families, with concentrated residence and coordinated resistance against the risk of adversity, has changed (Yang and Mukhopadhaya 2017). Another change is that family members have become more financially independent over the past 40 years of economic reform. Compared to couples who are used to living under a planned economy, with urbanization and marketization young couples now acquire financial support from the financial system and are less likely to share financial resources with parents or siblings (Jiang et al. 2012). Because of their financial independence and smaller size, families in today's China are less likely to acquire support from traditional family ties. As a result, the core family has become more essential as a source of support for families facing adversity ( $\mathrm{Li}$ et al. 2016).

Compared to urban families, livelihoods of rural families in today's China are more vulnerable, which entails that families have fewer resources and less capacity to improve their living conditions ( $\mathrm{Li}$ et al. 2012; Zhang 2016). Compared to urban families whose stability and sustainability is supported by companies, individual businesses, and urban welfare, rural families in China survive by constructing livelihoods that utilize resources from the natural environment, physical facilities, and social networks (Li et al. 2012). Compared to urban income, the income from rural livelihoods is lower, so that rural families have less money, less social capital, and fewer opportunities for education and social class improvement (Yang and Mukhopadhaya, 2017). This disadvantage is increasing with today's urbanization and rural-urban migration in China. In the last 40 years, rural Chinese families have decreased their dependence on natural capital replacing this with urban labor income from rural-urban migration, which has become the major source of income for rural families (Li et al. 2012). However, most families fail to improve their resilience because they give up traditional rural resources and rely instead on labor income, which is also needed for survival in the urbanized rural society (Wang and Tian 2014). In addition, rural families in today's China have hollowed out due to rural-urban migration. In traditional rural society without frequent migration, labor from within the extended family, as well as local social ties, have been important contributors to resilience when families struggle with natural disasters and social transitions (Chen and Korinek 2010). In today's China, however, rural families obtain less resilience from family members and local social ties because young rural residents usually migrate to cities (Wang and Tian 2014).

Studies on family resilience have concluded that resilience comes from family resources, including economic, social, and human (Wen and Hanley 2015; Taylor and Distelberg 2016; Vesely et al. 2017). Assessment of rural family resources incorporates sustainable livelihoods including natural capital, physical capital, social capital, financial capital, and human capital into an index of family resources that can contribute to the family's survival (Li et al. 2012). First, even though rural families are less likely to increase their income from natural resources, such as farmland, these resources still supply much of their basic food needs since income from urban labor of rural migrants is unstable (Chen and Korinek 2010). Second, housing conditions and facilities reflect how rural families may take advantage of physical resources to be resilient when confronted with floods, earthquakes, and medical problems (Abbas et al. 2018; Zhang 2016; Jiang et al. 2012). Third, rural families that have members who migrated have fewer social ties to their local rural communities but rarely form social ties with the urban communities; they still use rural social relationships as key supports when they need help (Wen and Hanley 2015). The other two capitals that are essential for rural families to survive are financial and human resource capitals. In urbanized and industrialized rural society, families significantly increase savings because 
they not only need funds for food and housing but also for improving their children's education, their living status, and the value of their individual labor (Chen and Korinek 2010). With respect to human capital, even though rural families have fewer people in residence and are less resilient in the face of sudden accidents, family members must still be relied on because there are fewer public services available in rural communities (Huang 2018). In summary, a rural family's sustainable livelihood may contribute to family resilience.

Previous studies have used scales to compare the status of family resilience across the globe, including China. These studies either assess a total score of resilience or score various dimensions of resilience, but ignore how basic family livelihoods affect the distribution of dimensional scores, which may reveal how family resilience is distributed into categories (Kaya and Arici 2012; Rocchi et al. 2017; Chew and Haase 2016; Isaacs et al. 2018). Studies of China must consider the current rural context, in which rural families experience urbanization and migration, and may have different types of resilience due to significant changes in sustainable livelihoods ( $\mathrm{Li}$ et al. 2012). To tell a story of family resilience among families in rural China, the studies mentioned above do not have a theoretical framework or data that can reveal family resilience. It is difficult to describe precisely the status of family resilience without considering the possible forms or categories of resilience and the effects of sustainable livelihoods. This study explores the status of family resilience in rural China from the perspective of sustainable livelihoods. We use latent analysis to reveal how categorical differences in family resilience are related to sustainable livelihoods.

\section{Theoretical Framework}

\subsection{Resilience in Family Theories and the Situation in Rural China}

In ecology, "resilience" is defined as the capacity of natural ecosystems to adapt to new environments and recover their original status after an environmental perturbation (Rus et al. 2018). In social science, resilience is defined as the capacity of a social system to adjust and use available resources to adapt to social changes (Ungar 2011). Family studies allude to resilience in explaining family status under uncertainty and adversity. Family system theory posits that a family's risks originate from systematic factors in the family's circumstances, and the family has an intrinsic capacity to use resources from its structure and from the external environment to maintain sustainable development (Patterson 2002). According to family pressure theory, both fixed factors such as family size, structure, and function, and dynamic factors such as within- and between-family connections, are strongly related to the family's adaptability to uncertainty (McCubbin and Patterson 1983). Both fixed and dynamic factors within the family reflect how it can become resilient when confronted with difficulties. The theory of family adaptability and response takes the capacity of the family under adversity as a dynamic process in which the division of labor and individual psychological adaptability play a role (Vesely et al. 2017). From the perspective of the family's adaptability, its resilience depends on its development, which includes the level of confidence within the family, enhancement of their resources, improvement of family structure, and frequency of cooperation among family members (Vesely et al. 2017).

The most accepted theory that directly defines family resilience is due to Walsh (2003), who summarized family theories in the context of normal families and defined family resilience as a capacity that originates from the family's resources, its structure, and its internal 
connections (Walsh 2003). Compared to the theories of Patterson, McCubbin and Vesely mentioned above, Walsh's definition of family resilience not only reflects internal and external factors that relate to family resources, but also reflects fixed and dynamic factors that relate to family adaptability under uncertainty (Walsh 2007). Walsh's theory is widely used in studies of how normal families maintain resilience using both internal and external resources (Rocchi et al. 2017; Sixbey 2005; Chew and Haase 2016; Isaacs et al. 2018). In studies on China, however, family resilience is not considered as a systematic family function among the majority of families, but as family relationships that exist under specific circumstances such as families with teenagers, families in need of aging support, and families facing medical problems (Li et al. 2016; Chen et al. 2018; Huang 2018). Considering rural China's urbanization and migration, Walsh's theory is relevant to family resilience among families in rural China. For instance, relationships within rural families in today's China are less likely than in the past to resolve family problems without considering family resources or structure. With reduction in family income due to reduced family size and fewer family laborers, economic and financial resources have become more essential for rural families to cope with adversities such as unemployment, natural disasters, and medical expenses (Jiang et al. 2012). The reduction in family size and family labor also leads to new family structures, in which family members not only have connections with parents, children and siblings, but also have frequent reciprocal cooperation within their social networks (Wen and Hanley 2015). Furthermore, physical capital, such as houses and facilities for production, has been enriched, which helps the family be more resilient when they have troubles (Zhang 2016). This indicates that family resilience in rural China is not only a function of the within-family relationships, but also includes family resources, structures, and social relationships (Li et al. 2016; Wen and Hanley 2015).

\subsection{Measures of Family Resilience and Global Applicability}

Measures of family resilience can be either objective or subjective: objective indexes reflect resources, such as natural and social capitals, which the family uses to respond to adversity without extra-familial support (Patterson 2002; Noor et al. 2014). There is no generally accepted objective measure, and studies have used measurements of family resilience according to the research context (Greeff et al. 2006; Card and Barnett 2015; Chen et al. 2018). Objective measures reveal the resource status of the family in a specific circumstance but are not guides for studying the majority of families in a time of significant social transition (Bhana and Bachoo et al. 2011). A subjective measure based on Walsh's theory, perceived family resilience (PFR), is a tool that not only reveals existing resource status but also shows how normal families react dynamically to adversity using available resources from family structures and social networks (Walsh 2007; Sixbey 2005; Kaya and Arici 2012; Chew and Haase 2016; Li et al. 2016; Isaacs 2018). PFR is defined as an individual's perceived attitudes towards his or her family, including that individual's attitude to family beliefs, availability of family's resources, flexibility of the family structure, and cooperativeness within the family (Ungar 2011; Walsh 2016). This resilience originates from the family's intrinsic capacity and availability of outside resources that allow family members to adapt to adversity while avoiding further negative outcomes (Coyle et al. 2009). PFR not only reflects what family members perceive about their family, but also reflects what they perceive outside the family (Walsh 2016).

Since factors contributing to PFR reflect multiple dimensions of family resilience, it is necessary to use multi-dimensional tools to study PFR quantitatively (Walsh 2007). Sixbey 
(2005) designed family resilience assessment scales (FRAS) that have a well-accepted total reliability of 0.96 for Cronbach's alpha. The dimensional reliability of subscales are 0.96 for family communication and problem solving, 0.85 for utilizing social and economic resources, 0.85 for maintaining a positive outlook, 0.70 for family connectedness, and 0.74 for making meaning of adversity (Sixbey 2005). FRAS have been applied in studies of various disadvantaged families and extended to 54 items, including "family communication and problem solving", "utilizing social and economic resources", "maintaining a positive outlook", "family inter-connectedness", "family spirituality", and "the ability to make meaning of adversity" (Windl et al. 2011). Scholars have revised the original FRAS to meet specific assumptions and apply to specific contexts. "The flexibility of the family" is included to measure how resilient the family structure is (Chew and Haase 2016). Another added dimension is "the obligation and the division of labor within the family", which reflects a family's collective effectiveness when its members cooperate (Chew and Haase 2016; Li et al. 2016). In addition, "cooperation among family members to reach a goal" reveals the status of within-family connections, by which even involuntary cooperation among family members may occur (Walsh 2016). The native versions of FRAS have proved to be reliable and valid in studies of Europe, Asia-Pacific, and Africa. In a study using a Turkish version of FRAS, the total Cronbach's alpha was 0.92 and the confirmatory factor analysis (CFA) results (root mean square error of approximation $($ RMSEA) $=0.058$, comparative fit index $(\mathrm{CFI})=0.92$, goodness of fit index $(\mathrm{GFI})=0.93$, standardized root mean square residual $($ SRMR $)=0.066)$ ) confirmed that the structure of subscales in the Turkish version was consistent with the original FRAS (Kaya and Arici 2012). Based on the English FRAS, the Italian FRAS achieved acceptable total Cronbach's $\alpha$ (0.946) (Rocchi et al. 2017). The CFA results in Rocchi et al.'s study also suggest that the structure of subscales in the Italian version was consistent with the original FRAS (RMSEA $=0.07, \mathrm{CFI}=0.96$, $\mathrm{GFI}=0.87, \mathrm{SRMR}=0.05)$. The Singapore FRAS, which is a version revised according to the social context of diverse ethnic families, achieved a total Cronbach's alpha of 0.92 and the subscale Cronbach's alphas ranged from 0.93 to 0.97 (Chew and Haase 2016). Isaacs et al. (2018) designed scales that showed how family beliefs and functions changed during the social transition in South Africa. This South African version of FRAS had a total Cronbach's alpha of 0.93 and the reliabilities of subscales were also acceptable (Cronbach's alpha was 0.97 for family communication and problem solving, 0.88 for utilizing social and economic resources, 0.86 for maintaining a positive look, 0.75 for making meaning of adversity), except for family connectedness, 0.38 (Isaacs et al. 2018).

Unlike the countries mentioned above that have Western culture, rural families in China live in a typical Eastern culture, where families are less religious and are more dependent on family beliefs and close family relations (Jiang et al. 2012; Huang 2018). Li et al. (2016) produced a short version of the Chinese FRAS with a total Cronbach's alpha of 0.95 . In this study, family spirituality related to religion was first removed from the original scales because religions have not been important to families since 1949 ( $\mathrm{Li}$ et al. 2016). Li et al. (2016) also introduced the Chinese version of the family assessment device (FAD), the perceived social support scale (PSSS), and the ten-item Connor-Davidson resilience scale (CD-RISC10) as corrections to the original FRAS. The results indicate that contents of some items overlapped when FRAS was tested among urban students and 34 items were retained to reflect family belief and communication (Cronbach's alpha $=0.94)$, utilizing social resources (Cronbach's alpha $=0.50$ ), maintaining a positive look and making meaning of adversity (Cronbach's alpha $=0.84)(\mathrm{Li}$ et al. 2016). However, no Chinese version of FRAS has been tested among families in rural China. 


\subsection{Impacts of Sustainable Livelihoods on Rural Families in China}

According to the Department for International Development's (DFID) in the UK, rural families acquire resources from their natural, physical, social, financial, and human capitals to survive and develop under natural and social adversity (Colombo et al. 2018). Compared to urban families, who have more wealth and more access to public services, rural families are more vulnerable under adversity because of uncertainty in the natural resources that are intrinsic to an agricultural economy (Baffoe and Matsuda, 2018). First, even though the majority of rural families are involved in migration, agriculture is still their major source of livelihood (Liu and Xu 2016). Rapid urbanization leads to reduction in the natural capital of farmlands and decrease in agricultural human resources, which reduce the amount of reliable resources and have negative impacts on rural families (Liu and Xu 2016). Second, physical capital, such as real estate and family facilities, is important to rural families experiencing sudden adversity. For instance, rural families experiencing medical problems are more resilient in terms of acquiring additional supportive resources if they have more family facilities (Chen et al. 2012). Another example is that rural families with more production facilities, such as harvesters and tractors, are not only more efficient in making money from farmlands, but also have more chance to supply commercial harvest services with their facilities and consequently have more money that can be saved for contingencies (Liu and Xu 2016). Third, social capital represents rural families' support from their social networks. Families with fewer friends have less chance to access such support (Ding et al. 2018). Additionally, both financial and human capital reflect the economic capacity for rural families to survive in adversity, especially when they experience natural disasters or recession (Wu et al. 2018; Li et al. 2012). Thus, sustainable livelihoods supply the available capitals that a family can use to be resilient, and then survive and develop during unexpected difficulties (Raffaelli et al. 2012).

\subsection{Limitations of Unclassified PFR Considering the Impacts of Sustainable Livelihoods on Rural Families}

Existing studies define PFR as the driving force for families to get through difficulties. They focus on the relations between specific dimensions of PFR and family survival issues. For instance, frequent communication and cooperation among family members improve adaptability to misfortune, and hence enhance the family's long-term well-being (Chen et al. 2018). In a study addressing families with illness, positive family belief and strong connections among family members were shown to help sick members cope with mental stress (Taylor and Disterlberg 2016). Also, optimism within a family increases its resistance to sudden crises and maintains its stability (Wen and Hanley 2015). Griffith (2005) found families in southern Europe that suffer from medical adversity kept daily life normal by increasing communication among family members, and families recover much faster from adversity if they have positive attitudes toward the family's future. Notably, families that experience frequent uncertainties, such as military families, are more likely to build positive family beliefs by which they recognize the bright side of negative outcomes and become resilient (Mancini et al. 2018). However, the studies mentioned above all focus on the impacts of family resilience on family survival but do not address empirically from where family resilience came. 
From Walsh's theory and previous studies, it is unclear how family factors, including belief system, communication, and pattern of organization, produce the power that makes families resilient. In discussions about uncertainties among rural families in China, the circumstances vary so rapidly that the family's structure, resource, and relationships change continually (Ding et al. 2018). The summary of sustainable livelihoods mentioned above indicates that whether rural families in China have resilience under social uncertainty probably depends on their sustainable livelihoods. Considering conditions in rural China, however, previous studies that use the total PFR score offer limited information as to how sustainable livelihoods lead rural families to resilience. First, rural families may have more resources due to an increase in labor income and growth of social networks during urbanization, whereas within-family connections, such as between adult children and aging parents or among siblings, may decrease because urban individuals are less likely to share information with families or communicate with relatives when they are busy with jobs (Jiang et al. 2012). However, rural families in less urbanized communities are still short of resources but usually keep the tradition of having a large family and strong connections among family members (Wang et al. 2020). They may have similar overall PFR score because families with fewer within-family connections may have more resources and families with less resources may have more within-family connections (Kaya and Arici 2012; Chew and Haase 2016; Rocchi et al. 2017; Isaacs, 2018). Second, assessing family resilience among rural families by comparing the overall PFR score may be misleading due to the effects of sustainable livelihoods. Even though rural families in China may have similar overall PFR scores because of their similar financial structure with a high saving rate and a low investment rate, they usually have different strategies that produce different degrees of resilience (Wang and Tian 2014). Thus, exploring characteristics of PFR by latent analysis may be appropriate for measuring family resilience in rural China's context of sustainable livelihoods (Henry and Muthen 2010).

According to Muthen's theory on exploring characteristic features, latent profile analysis (LPA) using PFR is suitable for identifying categories of family resilience and for estimating the contribution of each category to show the current status of family resilience in rural China (Nylund et al. 2007). Furthermore, regression mixture modeling (RMM) based on LPA may accurately estimate the impacts of sustainable livelihoods on family resilience (Muthen and Asparouhov 2009).

The present study assesses what individual family members perceive about their family's resilience based on Walsh's theory of family resilience and PFR. Compared to existing studies, the present study does not limit the PFR to be an overall score across families but uses the LPA method to partition PFR into different categories. The proportion of each category of PFR in the total sample is estimated to identify the features of family resilience. We also explore potential causal connections between sustainable livelihoods and features of PFR to reveal how family capitals in rural China affect rural family resilience.

\subsection{Data and Methodology}

\subsection{Data Collection}

Our data come from a self-reporting survey (the 2018 Hubei Multilevel Survey of Family Development and Governance in Urbanization), in which the livelihoods and living strategies at the family level and individuals' behaviors and perceptions about 
family resilience at the individual level were included. This two-week survey was implemented in August 2018 and was supported by the Family Development Department of the National Health Commission of China to explore how rural families survive and develop under rural China's urbanization and migration. In China's citizen registration system, rural identity has been eliminated since 2014. However, there is a long tradition that differentiates rural from urban citizens: people are still considered as rural residents if their homes are located in agricultural villages or if they live in urbanized rural towns (Wang and Tian 2014). Considering the purpose of this survey and the impacts of urbanization and migration, both rural families in agricultural villages and those in urbanized rural towns are included in the sampling.

The population of Hubei province is 59,020,000 (2017), distributed in thirteen cities and 61 counties. The sampling strategy was as follows: to sample rural families, counties were selected as sampling sites since the rural population is concentrated in counties instead of cities in China (Liu and Xu 2016). Two counties, including one that represents those with a huge population and another that represents those with a small population, were suggested by the local Family Development Department of the Health Commission of Hubei to make sure that the final rural samples represented the general situation in rural Hubei. H county (population 1,007,000; 2017) was randomly selected as a sampling site with a population over one million. Among sixteen towns in $\mathrm{H}$ county, twelve that rely mainly on agriculture were selected because the rural households in these are more concentrated (Awuah et al. 2017). In each town, only one community was randomly selected because of the homogeneity among such villages in rural China (Wu et al. 2018). J county (population 450,000, 2017) was randomly selected as a sampling site with population below one million. Three of the eight towns that were most urbanized in $\mathbf{J}$ county were selected to represent the general rate of urbanization. One of the selected towns still relies partially on agriculture and the other two rely mainly on non-agricultural industry. While only one community was selected in each town of $\mathrm{H}$ county, the sampling process in J county was as follows: two communities were randomly selected in the agricultural towns, where the distribution of households is scattered, to make sure the survey would include enough households; in each of the two non-agricultural towns, the survey team was advised by the local governor to increase the number of communities because rural residents with non-agricultural jobs are difficult to find; as a result three communities were randomly selected. The sampling sites are presented in Fig. 1.

Fig. 1 Sampling sites in Hubei province, China Source National Geomatics Center of China

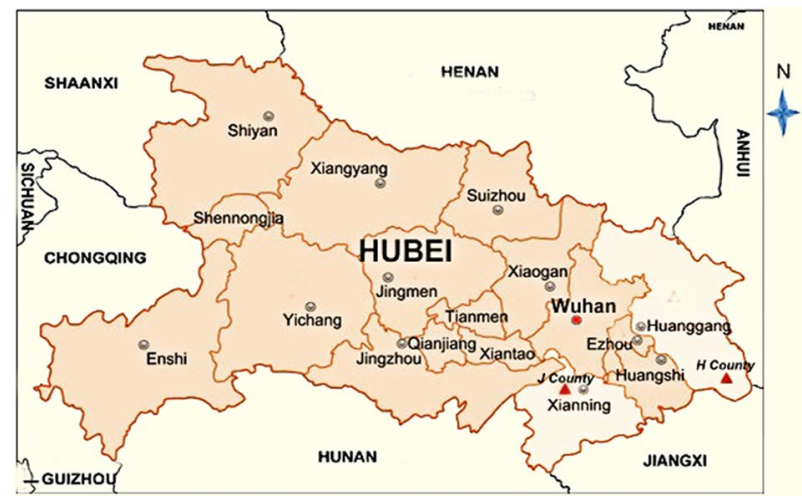


In both counties, fifteen households were randomly selected from each community. In each household, two individuals, including one who was responsible for family decisions and another family member, were invited to participate in the survey. Before the survey, individuals from each household were asked about who was responsible for family decisions. Identifying this person was important because this person usually plays core roles, including financial management and decision-making (Wu et al. 2018). This individual answered both a family questionnaire that reflects the family's resources and an individual questionnaire that reflects individual behaviors and perceptions. The other respondent, who was just another family member, only answered the individual questionnaire. Some families had more than two people participating in the questionnaire, and the additional person was another family member who only answered the individual questionnaire. Some families had only one person who answered both family and individual questionnaires.

In each community, respondents were invited to public spaces such as meeting rooms and squares to answer paper questionnaires. Considering China's tradition that rural people frequently hide or underreport their family assets (Wang and Tian 2014; Wu et al. 2018), the research team explained the purpose and anonymity of the investigation, and promised to protect family confidentiality. Also, the local community officers were required to leave to ensure this confidentiality. The final database included 702 samples of households and 1032 samples of individuals. Since natural capital is one of the key contributors to sustainable livelihoods in this study, samples from urbanized rural towns, who were no longer relying on agricultural incomes, were deleted from the total sample in order to make sure that the target samples were rural families with sustainable livelihoods. Finally, 370 households with 550 individuals were retained in this study. Detailed information about the households is presented in the Appendix Table 6. Of the 550 individuals, $48 \%$ were males; 94.4\% were married and $97 \%$ of those married had children; ages ranged from 16 to 73 and averaged 39.8; education duration (years) ranged from 0 to 17 and averaged 8 (junior middle school); personal income (yuan) ranged from 0 to 360,000 and averaged 22,471 ; $82.88 \%$ lived in agricultural villages and $17.12 \%$ lived in urbanized rural towns; $80.73 \%$ had rural-urban migration history and $97.63 \%$ had siblings.

\subsection{Measures of Sustainable Livelihoods}

Measures of sustainable livelihoods are included in the family questionnaire, which was answered by the respondent with family-decision responsibility within the household. Five indexes were used to define sustainable livelihood: natural capital $\left(\mathrm{X}_{1}\right)$, physical capital $\left(\mathrm{X}_{2}\right)$, social capital $\left(\mathrm{X}_{3}\right)$, financial capital $\left(\mathrm{X}_{4}\right)$, and human capital $\left(\mathrm{X}_{5}\right)$. The family's natural capital was measured by (1) annual agricultural income $\left(\mathrm{X}_{11}\right)$ and (2) acreage of farmland/water $\left(\mathrm{X}_{12}\right)$. Both items reflect the family's survival independent of markets or any commercial business. The family's received rents from farmland were included to reflect profits from natural resources. Physical capital was measured by (1) the area of the house $\left(\mathrm{X}_{21}\right),(2)$ rents from the house $\left(\mathrm{X}_{22}\right)$, and (3) possession of family facilities such as a tractor, harvester machine, motorcycle, car, and cattle $\left(\mathrm{X}_{23}\right)$. Social capital was measured by the number of the family's reliable relationships. Compared to urban families that rely mainly on social networks in communities and workplaces, families in rural China mainly acquire social support from their communities and relatives ( $\mathrm{Li}$ et al. 2012). We therefore use three measures of social capital: (1) the total number of the family's reliable relationships $\left(\mathrm{X}_{31}\right)$; (2) the number of the family's reliable relationships within the community $\left(\mathrm{X}_{32}\right)$; (3) the number of the family's reliable relationships with relatives $\left(\mathrm{X}_{33}\right)$. Financial 
capital is measured by (1) the proportion of the family's income that is banked $\left(\mathrm{X}_{41}\right),(2)$ the family's available money for investment $\left(\mathrm{X}_{42}\right)$, and (3) the family's financial arrears $\left(\mathrm{X}_{43}\right)$. The first two reflect the family's assets that are essential for the family to be resilient. The value of the third is negative in the calculation because it constitutes a family's financial disadvantage and decreases the family's resilience. Human capital reflects the family's human resources in agriculture and market commerce. We use (1) the number of family members in agriculture $\left(\mathrm{X}_{51}\right),(2)$ the number of family members who are migrant workers $\left(\mathrm{X}_{52}\right)$, and (3) the number of family members aged from 16 to 65 who are legal workers $\left(\mathrm{X}_{53}\right)$ to measure human resources. The values for each capital are aggregated to form a total score that reflects each capital (Ding et al. 2018). A standardization is first required to transform the original value of each item to take a value from 0 to 1 according to $X_{i j}^{\prime}=\left(X_{i j}\right.$ $\left.-X_{\mathrm{ijmin}}\right) /\left(X_{\mathrm{ijmax}}-X_{\mathrm{ijmin}}\right)\left(\right.$ Pandey et al. 2017). Then $X_{\mathrm{i}}$ in Table $1(\mathrm{i}=1,2, \ldots \ldots .5)$ are the final values of the five capitals, which are calculated as weighted averages of the $X_{i j}^{\prime}$ values from the Appendix Table 6 (Liu and Xu 2016). Detailed calculations of sustainable livelihoods are presented in the Appendix Table 6.

\subsection{Measurements and Statistical Strategy for Perceived Family Resilience}

PFR in this study was measured by scales for the individual questionnaire, which was answered by each respondent from each household. At the surface level, family belief system, family communication and family pattern of organization are basic concepts (Walsh 2003). For the second step, seven key indexes of PFR were based on Western scales: "maintaining a positive outlook" (MPO), "making meaning of adversity" (MMA), "cooperate to reach a family goal" (CRG), "family inter-connection" (FIC), "family flexibility" (FF), "family utilization of social resources" (FUSR) and "family structure and obligation" (SO) (Sixbey 2005; Walsh 2016; Chaw and Haase 2016). At a deep level, 34 items with 5-point Likert scales from the Chinese FRAS are used (Li et al. 2016). Li et al. integrated 34 items into family belief and communication, utilizing social resources, maintaining a positive look and making meaning of adversity. However, this study integrates the 34 items into the seven key indexes mentioned above based on the original, European and Asia-Pacific FRAS (Sixbey 2005; Rocchi et al. 2017; Chew and Haase 2016). Each of the seven key indexes is the mean value of these items. Table 2 presents seven key indexes for PFR and related items. The total reliability achieves good acceptance (Cronbach's alpha $=0.930$ ) and the reliability Cronbach's alpha of the seven key indexes are 0.814 for MPO, 0.728 for MMA, 0.711 for CRG, 0.646 for FIC, 0.674 for FF, 0.629 for FUSR and 0.750 for SO.

Table 1 Measurements of sustainable livelihood $(\mathrm{N}=370$ households)

\begin{tabular}{llll}
\hline $\begin{array}{l}\text { Sustainable livelihood } \\
\text { family level) }\end{array}$ & Mean & SD & Min/Max \\
\hline Natural capital $\left(\mathrm{X}_{1}\right)$ & 0.032 & 0.043 & $0 / 0.317$ \\
Physical capital $\left(\mathrm{X}_{2}\right)$ & 0.179 & 0.080 & $0.006 / 0.638$ \\
Social capital $\left(\mathrm{X}_{3}\right)$ & 0.080 & 0.077 & $0 / 0.615$ \\
Financial capital $\left(\mathrm{X}_{4}\right)$ & 0.036 & 0.062 & $-0.156 / 0.328$ \\
Human capital $\left(\mathrm{X}_{5}\right)$ & 0.277 & 0.121 & $0 / 0.750$ \\
\hline
\end{tabular}

Source 2018 Hubei Survey on family development and governance in urbanization 


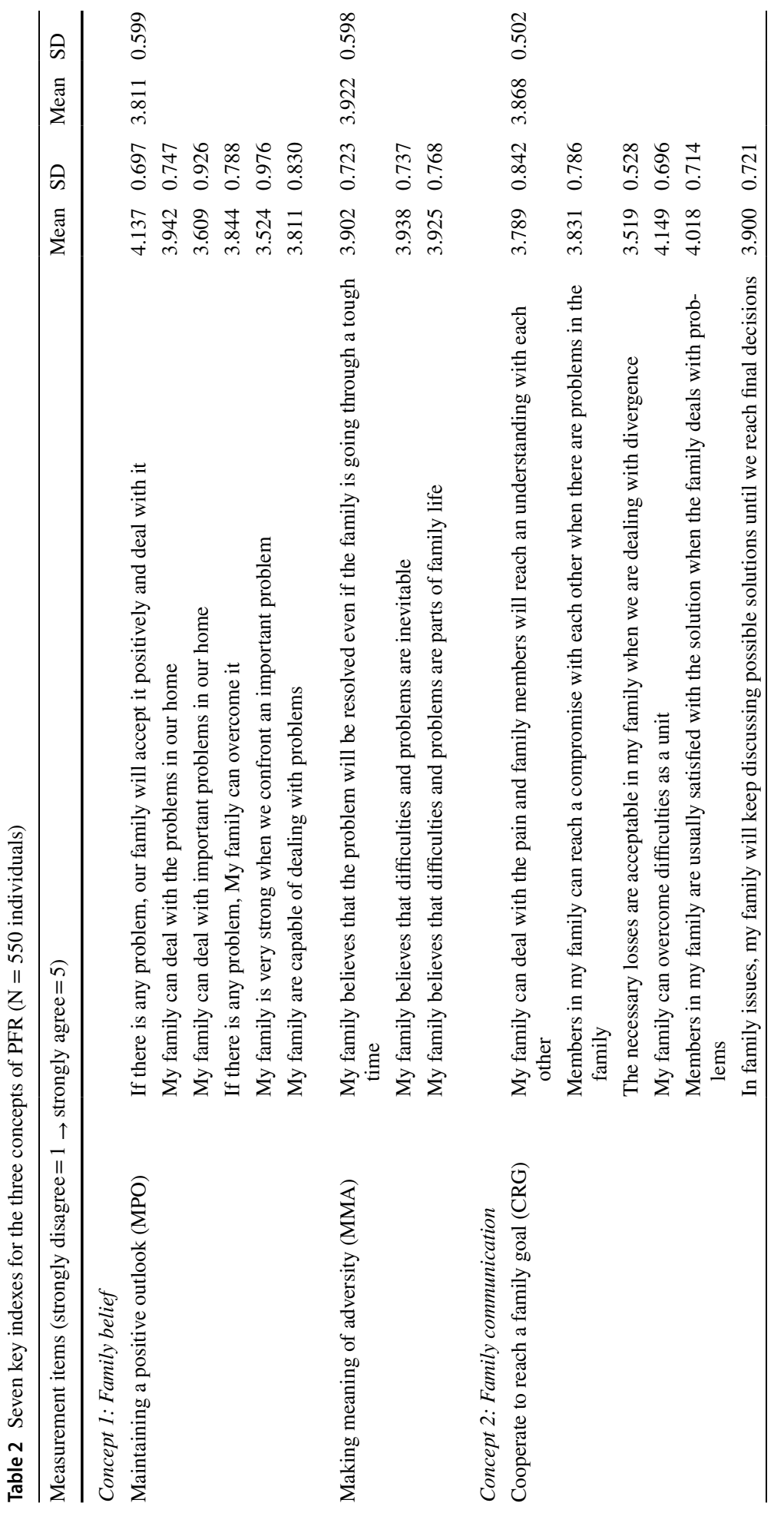




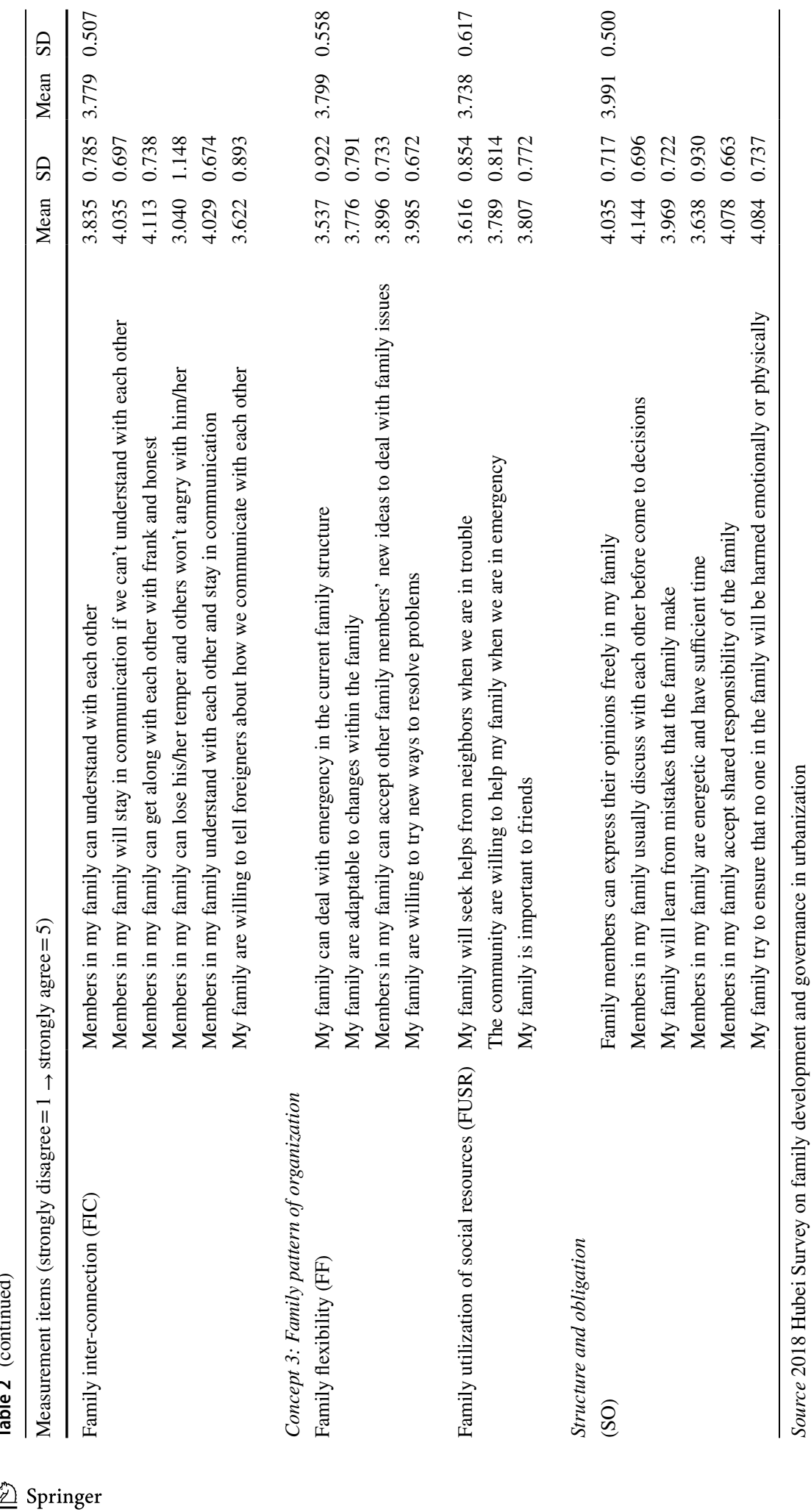


We use latent profile analysis (LPA) to identify categories of PFR. LPA classifies samples into categories and estimates the probability of each category; the score of each index within each group is also estimated (Muthén and Muthén 2017). In LPA, first only one category is assumed and the statistical parameters are estimated. Then two categories are assumed and the statistical parameters are estimated again. This process continues until an optimal classification model is found (Clark et al. 2015), according to the values of AIC (Akaike information criterion), BIC (Bayesian information criterion) and ABIC (samplesize-adjusted Bayesian information criterion) (Lee et al. 2014). If the values of AIC, BIC, and ABIC in the model with two categories are significantly lower than those in the model of one category, then the former model is regarded as better than the latter (Muthén and Asparouhov 2009). This process continues until the values of AIC, BIC, and ABIC in the last model are not significantly lower than those in the previous model; then the previous model is retained and regarded as the optimal classification (Nylund et al. 2007). Another index for optimal classification model is entropy, which ranges from 0 to 1 . The closer entropy is to 1, the more reliable the model (Vermunt 2008). We use regression mixture modeling (RMM) to assess causal connections between the identified category of individual PFR and covariates. With a multilevel nested survey, we use multilevel regression mixture modeling (MRMM), where both individual-level and family-level variables are included. In this study using MRMM, the identified category of individual PFR (variables from the individual questionnaire) is the dependent variable and both sustainable livelihoods (variables from the family questionnaire) and individual variables (gender, marriage and age from the individual questionnaire) are included as covariates (Henry and Muthén 2010). We use M-plus to apply LPA and MRMM.

\section{Results and Discussion}

\subsection{Classification and Categorical Features of PFR}

Table 3 gives LPA results assuming from one to four categories. The only variables in LPA are the seven indexes of PFR, including "maintaining a positive outlook" (MPO), "making meaning of adversity" (MMA), "cooperate to reach a family goal" (CRG), "family inter-connection" (FIC), "family flexibility" (FF), "family utilization of social resources" (FUSR) and "family structure and obligation" (SO). Compared to the model with two classes, the model with three classes shows a significant decrease of AIC, BIC, and ABIC. Entropy in the model with three classes is 0.903 , the highest among all models. Compared

Table 3 Process of latent profile analysis

\begin{tabular}{|c|c|c|c|c|}
\hline \multicolumn{5}{|c|}{ Number of PFR categories } \\
\hline Model & 1 class model & 2 classes model & 3 classes model & 4 classes model \\
\hline AIC & 6374.223 & 5335.599 & 4700.535 & 4505.557 \\
\hline $\mathrm{BIC}$ & 6434.562 & 5430.418 & 4829.832 & 4669.334 \\
\hline ABIC & 6390.120 & 5360.580 & 4734.599 & 4548.706 \\
\hline Entropy & - & 0.886 & 0.903 & 0.881 \\
\hline
\end{tabular}

Source 2018 Hubei Survey on family development and governance in urbanization 
to the model with three classes, the model with four has a less significant decrease of AIC, BIC, and ABIC. Entropy in the model of four categories is also lower than 0.903. According to the statistical rule of LPA (Muthén and Muthén 2017), the model with three classes of PFR is the most acceptable in Table 3.

LPA gives the estimated scores of the seven indexes ("MPO", "MMA", "CRG", "FIC", "FF", "FUSR" and "SO") in each category, and the probability of each category is estimated. Table 3 shows that the model with three categories of PFR is the most acceptable. Since it is hard to see the qualitative features of these, we make one more standardized calculation within each category: the estimated score of each index subtracts the average of the total scores of the seven indexes, and the final score for each index of PFR gives features of each category of PFR shown in Fig. 2.

Figure 2 shows "family structure and obligation" (SO) and "making meaning of adversity" (MMA) in each category giving similar results: having the highest and second highest scores among the seven indexes of PFR. This agrees with a previous finding that no matter how rural families change, neither basic family obligations nor family division of labor change significantly (Huang 2018). Interestingly, the Singaporean FRAS in the study by Chew and Haase (2016) had contrary results, in which family roles and obligations are strongly self-esteemed instead of immutable. The score for MMA indicates again that Chinese rural families during social transition can retain stable connections among family

\section{Latent profile solution}

Standardized score of the 7 indexes

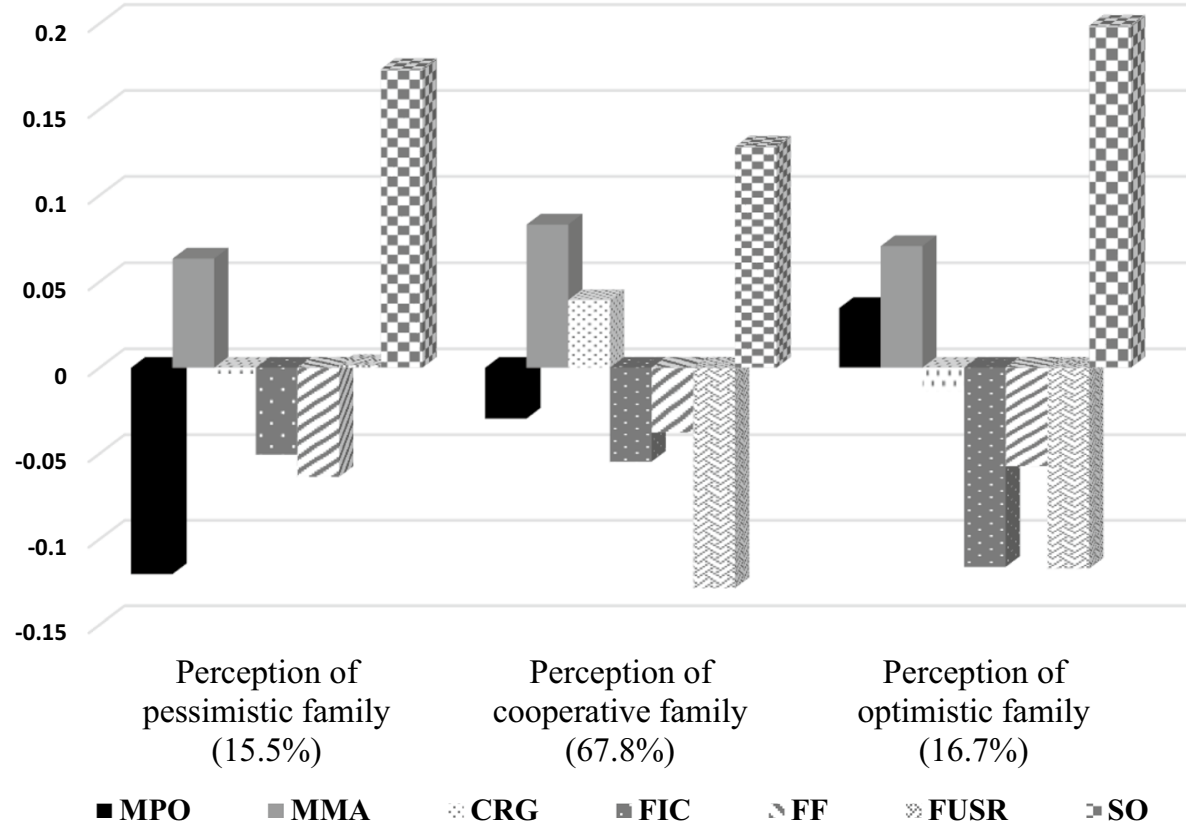

Fig. 2 Final classification of perceived family resilience (PFR) Source 2018 Hubei Survey on family development and governance in urbanization. Note MPO, maintaining a positive outlook; MMA, making meaning of adversity, CRG, cooperate to reach a family goal, FIC, family inter-connection, FF, family flexibility, FUSR, family utilization of social resources, SO, family structure and obligation 
members, which is called "self-adaptability", and reflects how the family spontaneously adapts to negative outcomes (Chen and Korinek 2010). Similarly, the Singaporean FRAS results, which includes some Chinese families, also suggest that Eastern-culture families can acquire resilience by positive beliefs, such as always looking on the bright side (Chew and Haase 2016). The differences among the remaining indexes separate the three categories from each other. The first group is the first category of the rightmost group in Fig. 2, in which "maintaining a positive outlook" (MPO), reflecting optimism in the family, has the highest score. We name this category "perception of optimistic family", whose categorical probability is 0.167 , which means $16.7 \%$ of the 550 samples (Muthén and Asparouhov 2009). The European study found that families using strong family belief to produce resilience usually focus on shared beliefs and supports instead of optimistic beliefs (Rocchi et al. 2017). The other two categories have no strong feature of optimism. Compared to the first group on the left, the middle group has the highest score in "cooperate to reach a family goal" (CRG). We name this "perception of cooperative family", and it makes up $67.8 \%$ of the samples. Interestingly, for African rural families that are religiously oriented, the majority of people acquire family resilience from family spirituality instead of family cooperation (Isaacs et al. 2018). The left-most group has the lowest score in "maintaining a positive outlook" (MPO): these people are more likely to have pessimistic perceptions of their families. We name this group "perception of pessimistic family"; it covers $15.5 \%$ of individual samples. For this classification of PFR, the majority of rural families' resilience is perceived as a cooperative mechanism, whereas fewer rural families perceive resilience in terms of optimism and pessimism.

The feature of the perception of optimistic family in this study is positive adaptability to adversity. This helps families adapt to new environments and reduces negative effects (Griffith et al. 2005). The scores of "family inter-connection" (FIC) and "family utilization of social resources" (FUSR) in optimistic families, however, are significantly lower than other indexes within this group, indicating that optimistic families may neglect cooperation between family members (Chen et al. 2018). Since families with larger social networks adapt more easily to adversity (Chen et al. 2012), people from optimistic families may have difficulty acquiring outside support due to the reduced attention they pay to utilization of social resources. People from cooperative families deal with uncertainties by using more cooperation within their family. Compared to other families, they have stronger connections among family members and more cooperation, both of which are advantageous for the family to react quickly (Chew and Haase 2016). However, people from cooperative families may have difficulty acquiring outside support due to their reduced utilization of social resources. The last category is the perception of pessimistic family, whose "maintaining a positive outlook" (MPO) is the lowest. Pessimistic families have the weakest connections among family members and the lowest flexibility. This agrees with the finding that pessimistic families lack family communication without which family members are inflexible in responding to adversity (Wen and Hanley 2015).

\subsection{Resilience and Adaptability Among Categorical Groups of PFR}

In this study, LPA estimates the overall score of the seven indexes of PFR in each category as an additional criterion for comparing family resilience among classes of PFR (Muthen and Asparouhov 2009). Compared to the overall score of PFR that directly defines family resilience for all samples, the scores of the seven indexes of PFR in each category not only reflect family resilience within that category but also allow 
comparison of resilience status among different categories (Clark et al. 2015). According to Fig. 2 and LPA estimation, the overall scores of the seven indexes of PFR are $22.001,26.957$, and 31.199 for the perceptions of pessimistic family, cooperative family, and optimistic family, respectively. This suggests that optimistic families may have the highest resilience, whereas cooperative and pessimistic families may have medium and lowest resilience, respectively (Kaya and Arici 2012; Chew and Haase 2016; Li et ail. 2016; Isaacs 2018). As family resilience in this study is defined as a family's capacity to adapt, the higher the family resilience, the greater is its adaptability. Table 4, which shows the connection between family resilience and adaptability, also shows how sustainable livelihoods are distributed across classes based on LPA results.

First, the natural capital scores in Table 4 show that rural families who have more resilience have more natural capital and will be more adaptable. Noting that the income structure in the labor market has been highly unstable since the financial crisis of 2018, rural families still regard farmlands as indispensable resources (Wang and Tian 2014). The case study in western rural China indicates that rural families, who are involved in seasonal agricultural activities, are more likely than those who fully quit agricultural activities to adapt to the shake-up in labor markets such as occurred in 2008 (Li et al. 2012). On the other hand, rural families who stop getting income from natural resources have occasional difficulties due to the unemployment in the market economy (Wu et al. 2018). Second, the physical capital scores in Table 4 show that rural families who have more resilience have more physical capital and will be more adaptive. Also, rural families can acquire extra money if they get rent from land or houses, and families who have more agricultural machines participate in commercial agricultural services and can make extra money as they adapt to the marketization of rural society (Zhang et al. 2015). By contrast, rural families with few available physical facilities usually live in poverty and become overwhelmed if anyone becomes sick or their children need higher education (Huang 2018; Yang and Xu 2019). Third, the social capital scores in Table 4 show that rural families who are more resilient have more social capital and will be more adaptive. Considering the incomplete public service system in rural China, families who have stable connections to friends and relatives can find support more readily if they are seeking help for aging relatives or in natural disasters (Jiang et al. 2012; Zhang 2016). However, rural families with fewer social relationships have to deal with uncertainties on their own and take longer to adapt to new difficulties (Wen 2015).

Table 4 Sustainable livelihood among groups of PFR

\begin{tabular}{llll}
\hline Sustainable livelihood & $\begin{array}{l}\text { Class 1 Perception of } \\
\text { pessimistic family(low } \\
\text { resilience) } \\
\text { Mean (S.E.) }\end{array}$ & $\begin{array}{l}\text { Class 2 Perception of } \\
\text { cooperative family } \\
\text { (medium resilience) } \\
\text { Mean (S.E.) }\end{array}$ & $\begin{array}{l}\text { Class 3 Perception of } \\
\text { optimistic family (high } \\
\text { resilience) } \\
\text { Mean (S.E.) }\end{array}$ \\
\hline Natural capital & $0.029(0.004)$ & $0.033(0.002)$ & $0.033(0.003)$ \\
Physical capital & $0.179(0.010)$ & $0.180(0.004)$ & $0.188(0.008)$ \\
Social capital & $0.065(0.006)$ & $0.082(0.004)$ & $0.097(0.010)$ \\
Financial capital & $0.038(0.007)$ & $0.036(0.003)$ & $0.031(0.006)$ \\
Human capital & $0.270(0.014)$ & $0.280(0.006)$ & $0.278(0.014)$ \\
\hline
\end{tabular}

Data: 2018 Hubei Survey on family development, resilience and governance in urbanization 
From the perspective of financial capital in Table 4, however, the connection between family resilience and family adaptability is the opposite of that expected; families with higher resilience are less likely to be financially adaptive. The financial capital index in this study mainly reflects the family's bank deposits and investment rate. However, this is probably consistent with the actual rural situation, in which rural families with less resilience are more likely to keep money on deposit to avoid uncertainties (Wang and Tian 2014). In a commercialized and marketized rural society like today's China, rural families will be less adaptive if they hesitate to use financial resources (Wu et al. 2018). From the perspective of human capital in Table 4, cooperative families with medium resilience have the most human capital, whereas optimistic families, i.e., those with the highest resilience, have medium human capital and pessimistic families with the lowest resilience have the lowest human capital. This is consistent with the finding that rural families with more laborers will adapt more rapidly and easily to medical contingencies, aging support, and sudden accidents (Zhang 2016; Yang and Mukhopadhaya 2017; Huang 2018).

\subsection{Multilevel Effects on Classification of Perceived Family Resilience}

Our final aim is to see whether family sustainable livelihoods have significant effects on the classification of PFR. Classifications defined by LPA at the individual level will be dependent categorical variables. Individual factors and family capitals are covariates in the analysis. We use the multilevel regression mixture model (MRMM), and the results are shown in Table 5, where the perception of the cooperative family is the reference category since the number of individual samples within this category is the largest in Fig. 2 (Muthén and Asparouhov

Table 5 Multilevel effects on latent profile solution

\begin{tabular}{|c|c|c|c|c|c|c|}
\hline \multirow{3}{*}{$\begin{array}{l}\text { zReference: Perception of } \\
\text { cooperative family }\end{array}$} & \multicolumn{3}{|l|}{ Model 1} & \multicolumn{3}{|l|}{ Model 2} \\
\hline & \multicolumn{3}{|c|}{ Perception of pessimistic family } & \multicolumn{3}{|c|}{ Perception of optimistic family } \\
\hline & $\beta$ & S.E & $\mathrm{p}$ & $\beta$ & S.E & $\mathrm{p}$ \\
\hline \multicolumn{7}{|l|}{ Individual level } \\
\hline Gender (Ref: male) & 0.462 & 0.001 & $0.000^{* * *}$ & -0.088 & 0.011 & $0.000^{* * *}$ \\
\hline Marriage (Ref: unmarried) & 0.273 & 0.007 & $0.000^{* * *}$ & -0.465 & 0.017 & $0.000^{* * *}$ \\
\hline Age & -0.024 & 0.000 & $0.000^{* * * *}$ & 0.066 & 0.001 & $0.000^{* * *}$ \\
\hline \multicolumn{7}{|l|}{ Family level } \\
\hline Natural capital & 3.001 & 0.009 & $0.000^{* * * *}$ & 3.645 & 0.127 & $0.000^{* * * *}$ \\
\hline Physical capital & -6.269 & 0.031 & $0.000^{* * * *}$ & -0.287 & 0.165 & $0.000^{* * * *}$ \\
\hline Social capital & 3.598 & 0.002 & $0.000^{* * *}$ & 2.126 & 0.158 & $0.000^{* * *}$ \\
\hline Financial capital & -0.640 & 0.006 & $0.000^{* * * *}$ & -1.514 & 0.012 & $0.000^{* * *}$ \\
\hline Human capital & -3.612 & 0.021 & $0.000^{* * *}$ & 4.213 & 0.046 & $0.000^{* * *}$ \\
\hline Intercept & 10.692 & 0.021 & $0.000^{* * *}$ & -21.816 & 0.305 & $0.000^{* * *}$ \\
\hline AIC & 2203.30 & & & & & \\
\hline $\mathrm{BIC}$ & 2369.67 & & - & & & \\
\hline $\mathrm{ABIC}$ & 2223.81 & & - & & & \\
\hline df & 46 & & - & & & \\
\hline Entropy & 0.910 & & - & & & \\
\hline
\end{tabular}

Source: 2018 Hubei Survey on family development and governance in urbanization $* \mathrm{p}<0.05, * * \mathrm{p}<0.01, * * * \mathrm{p}<0.001$ 
2009; Henry and Muthén 2010). At the individual level, gender, marriage and age are introduced into the regression as individual covariates. Social individual factors, including education and income, are not included in the regression since they may have endogenous effects with factors of sustainable livelihood at the family level (Ding et al. 2018).

The intercepts in models 1 and 2 indicate that family variables have significant effects on PFR. Perceptions of family structure, family roles and family relations are related to the family's economics, social networks and finance (Baffoe and Matsuda, 2018). Also, people may have different perceptions of family status due to their different contributions to the family's capitals (Griffith et al. 2005). Therefore, it is reasonable that the status of family resilience depends on family's sustainable livelihood.

Model 1 shows the comparison between the perceptions of cooperative and pessimistic families. At the individual level, women and married people are more likely to perceive family affairs pessimistically than cooperatively. Ding et al. (2018) suggested that compared to rural men, who usually have a higher frequency of migration, rural women are more likely to stay at home and are more frequently confronted with family uncertainties. This may be why rural women are more likely to perceive that their families show less adaptability to difficulties. Compared to unmarried residents in rural families, married residents more frequently experience problems when they seek resources for the family (Huang 2018). Compared to older people, younger people are more likely to perceive family affairs cooperatively than pessimistically. The reason is that younger residents are more likely to quit the agricultural economy and migrate, for which they need more support from the family (Chen and Korinek 2010).

Model 1 indicates that sustainable livelihoods have significant impacts on PFR. First, people from rural families with more natural and social capitals are more likely to have the perception of a pessimistic family than a cooperative family. More natural capital indicates a stronger dependence on the agricultural economy. Under urbanization and rural industrialization, rural families that are more dependent on natural capital are vulnerable and are likely to suffer from economic and social uncertainties in China's social transition (Li et al. 2012). Second, in rural China, families traditionally receive strong support from their social and family networks (Wen and Hanley 2015). However, rural families that maintain strong connections to rural social networks usually have fewer migrants and lack other social resources, without which they may have difficulty overcoming adversity.

Model 1 indicates that people from rural families with more physical, financial, and human capitals are more likely to have the perception of a cooperative family than a pessimistic family. A likely explanation is that during China's economic growth, most rural families have increased their material property, have acquired more financial resources, and have had more chances to send family members into the labor market (Chen et al. 2012; Wu et al. 2018). This suggests that rural families currently benefit from more family cooperation in dealing with uncertainty. More physical and financial capitals enables rural people to participate in diverse economic production, in which the division of family labor and cooperation within the family increase (Baffoe and Matsuda 2018; Chen and Korinek 2010).

Model 2 shows the comparison between the perceptions of a cooperative family and of an optimistic family. At the individual level, women and married people are more likely to perceive family affairs cooperatively than optimistically. Rural women are more likely than rural men to stay at home with their families ( $\mathrm{Li}$ et al. 2012). Without men's support, rural women may prefer family cooperation over optimism. Compared to the unmarried, married people usually play the roles of parents or supporters and prefer the cooperative mechanism within the family to improve the wellbeing of all family members ( $\mathrm{Li}$ et al. 2012). Members of the older generation are more likely to perceive family affairs cooperatively than 
optimistically. A reasonable explanation is that the older generation are more likely to make family decisions, and such decisions may require discussion and cooperation among family members (Chen et al. 2018).

Model 2 indicates again that sustainable livelihood has a significant impact on PFR. First, people from rural families with more natural, social, and human capitals are more likely to have an optimistic perception of family affairs than a cooperative one. The better status of these capitals indicates that rural families have more natural and social resources to improve the family wellbeing, and have more human support to deal with economic pressure, medical risk, and aging (Bhana and Bachoo 2011). Rural families with more natural, social, and human resources usually have better living conditions, which should induce optimistic attitudes within the family. However, people from families with more physical and financial capitals are more likely to have the perception of a cooperative family than an optimistic family. More physical capital indicates that rural families have more tangible properties, which are usually regarded as indirect economic resources (Colombo et al. 2018). Therefore, there will be more discussion about how to use physical capital. Financial resources lead rural families to have more investments, about which discussion and cooperation within families are likely to occur (Li et al. 2012).

\section{Conclusion}

This study contributes to family resilience research in two ways. First, we do not limit the focus to the overall PFR score but discuss the scores on scales of several dimensions of PFR. Compared to existing studies that define the resilient status for all family samples, this study introduces LPA to find categories of resilient families. Sustainable livelihoods, which are essential for rural families to survive, are first incorporated into Walsh's theory of family resilience to explore where and how family resilience originates in rural areas using the MRMM method. Ours is a pilot study for research that will focus on family resilience that incorporates basic natural and social resources. Second, we designed a Chinese version of FRAS for rural samples that shows how family resilience is distributed across families under rapidly changing sustainable livelihoods. Due to the complicated situation of urbanization and migration, as well as the correlation between family resilience and sustainable livelihoods, further research on rural families in China is suggested to consider the sensitivity of family resilience to family resource changes, especially in the more urbanized provinces on the east coast of China.

Three categories of PFR have been identified: perception by the optimistic family with the highest score of resilience, perception by the cooperative family with a medium score of resilience, and perception by the pessimistic family with the lowest score of resilience. People from optimistic families have the highest score for optimistic belief, which reflects their stronger psychological adaptability in response to difficulties. However, they have less communication within the family and are not likely to seek outside support. People from cooperative families can acquire family resilience by frequent cooperation within the family. People from pessimistic families have significantly negative attitudes toward adversity, fewer connections within the family, and less family flexibility. These contribute to low resilience, and the family in adversity will react slower and have less resources to mitigate risks. Pessimistic families are the most vulnerable group and are in need of outside support. Based on a comparison of sustainable livelihoods among the resilience categories of families, we suggest that rural families with more natural, physical, social and human resources are more likely to be resilient and therefore adaptive. 
Gender, marital status and age have significant effects on PFR. Rural men have more optimism than rural women in the evaluation of family resilience. Since rural men frequently migrate, their optimistic evaluation about their family's adaptability to adversity may be exaggerated. Patterns differ between rural families with less resilience and those with more resilience. In the comparison between families with less resilience, namely pessimistic and cooperative families, unmarried members perceive family cooperation because they are more likely to need family support to achieve their needs. In the comparison between families with more resilience, namely cooperative and optimistic families, married members perceive family cooperation because they are more likely to cooperate in making decisions using their dominant roles. In general, young people are more likely than older people to perceive family cooperation. This consistent effect of age indicates that compared to their elders, younger people will seek support from their families more frequently to achieve goals in school, the job market, and society.

Family resilience in rural China depends significantly on the status of the family's sustainable livelihood. Rural families with more natural and social capital will be less resilient. These capitals are traditional resources for resilience of rural families in an agricultural economy: rural families have a stable structure and a division of labor that produces resilience and have no need for frequent discussion. In the social transition, however, rural families have to deal with difficulties due to urbanization and rural industrialization, and today traditional natural resources and rural social networks are less able to support rural families' response to risks. Both natural and social capitals in rural communities are decreasing rapidly; families that are overly dependent on traditional rural resources will be less resilient in today's social transition.

Rural families with more physical and financial capitals will be more resilient. Better physical capital indicates more physical properties, from which rural families can derive extra resources when they deal with challenges, such as medical needs, marriage costs, and aging support. Greater financial capital indicates more family wealth, by which rural families will directly overcome risks and protect wellbeing. Unlike natural and social capitals, neither physical capital nor financial capital can be contributed by any single member. Instead, all family members have to cooperate to accumulate physical property and financial wealth. Even though rural families in modern China have less traditional resources from the agriculture economy, they are still capable of improving family resilience by resources from markets, industries, and new social networks.

Our sample is from a central Chinese province, which may not be representative of all of rural China. The sampling sites at the county level reflect only the average situation of counties in Hubei Province. Those countries with either rapid economic development in rapid urbanization or poverty in mountainous regions are not represented here.

Open Access This article is licensed under a Creative Commons Attribution 4.0 International License, which permits use, sharing, adaptation, distribution and reproduction in any medium or format, as long as you give appropriate credit to the original author(s) and the source, provide a link to the Creative Commons licence, and indicate if changes were made. The images or other third party material in this article are included in the article's Creative Commons licence, unless indicated otherwise in a credit line to the material. If material is not included in the article's Creative Commons licence and your intended use is not permitted by statutory regulation or exceeds the permitted use, you will need to obtain permission directly from the copyright holder. To view a copy of this licence, visit http://creativecommons.org/licenses/by/4.0/.

\section{Appendix}




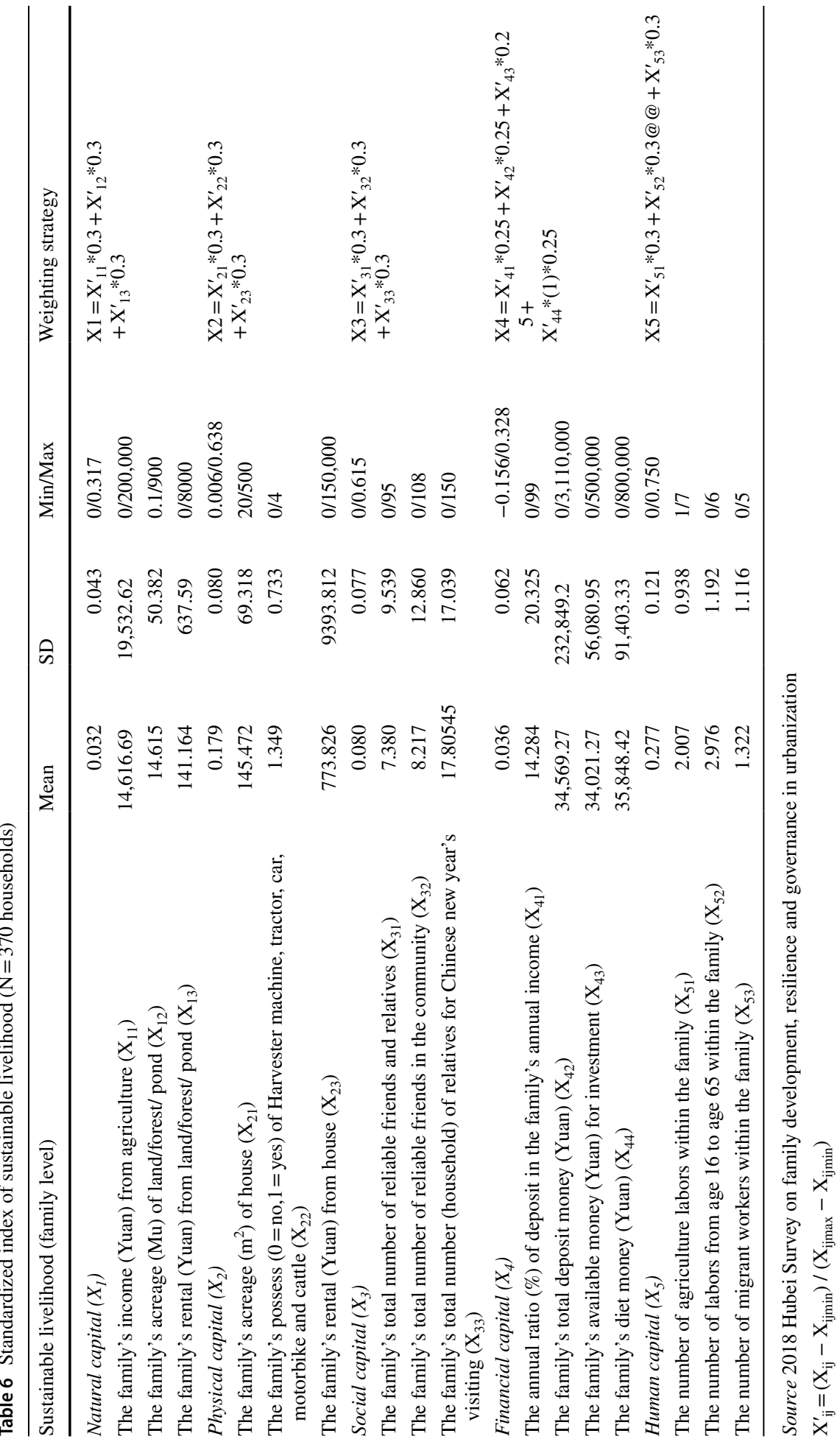




\section{References}

Abbas, A., Amjath-Babu, T. S., Kachele, H., Usman, M., Iqbal, M. A., Arshad, M., et al. (2018). Sustainable survival under climatic extremes: linking flood risk mitigation and coping with flood damages in rural Pakistan. Environmental Science and Pollution Research, 25, 32491-32505.

Awuah, R., Douglass, R., Agyepong, S., \& Kuwornu, E. (2017). An adaptive household sampling method for rural African communities. African Journal of Food, Agriculture, Nutrition and Development, 17(1), 11477-11496.

Baffoe, G., \& Matsuda, H. (2018). An empirical assessment of households livelihood vulnerability: The case of rural Ghana. Social Indicators Research, 140(3), 1225-1257.

Bhana, A., \& Bachoo, S. (2011). The determinants of family resilience among families in low and middle income contexts: A systematic literature review. South African Journal of Psychology, 41(2), 131-139.

Card, N. A., \& Barnett, M. A. (2015). Methodological considerations in studying individual and family resilience. Family Relations, 64(1), 120-133.

Chen, F. N., \& Korinek, K. (2010). Family life course transitions and rural household economy during China's market reform. Demography, 47(4), 963-987.

Chen, H. Y., Shivakoti, G., Zhu, T., \& Maddox, D. (2012). Livelihood sustainability and community based co-management of forest resources in China: changes and improvement. Environmental Management, 49(1), 219-228.

Chen, C. M., Du, B. F., Ho, C. L., Ou, W. J., Chang, Y. C., \& Chen, W. C. (2018). Perceived stress, parentadolescent/young adult communication, and family resilience among adolescents/young adults who have a parent with cancer in Taiwan: A longitudinal study. Cancer Nursing, 41(2), 100-108.

Chew, J., \& Haase, A. M. (2016). Psychometric properties of the family resilience assessment scale: A singaporean perspective. Epilepsy \& Behavior, 61, 112-119.

Clark, T. T., Salas-Wright, C. P., Vaughn, M. G., \& Whitfield, K. E. (2015). Everyday discrimination and mood and substance use disorders: A Latent profile analysis with African Americans and Caribbean Blacks. Addictive Behaviors, 40, 119-125.

Coyle, J. P., \& Nochajski, t., Maguin, E., Safyer, A., Dewit, D., \& Macdonald, S. . (2009). An exploratory study of the nature of family resilience in families affected by parental alcohol abuse. Journal of Family Issues, 30(2), 1606-1623.

Colombo, E., Romeo, F., Mattarolo, L., Barbieri, J., \& Morazzo, M. (2018). An impact evaluation framework based on sustainable livelihoods for energy development projects: An application to Ethiopia. Energy Research \& Social Science, 39, 78-92.

Ding, W. Q., Jimoh, S. O., Hou, Y., Hou, X. Y., \& Zhang, W. G. (2018). Influence of livelihood capitals on livelihood strategies of herdsmen in inner Mongolia. China. Sustainability, 10(9), 3325-3341.

Gallopín, G. C. (2006). Linkages between vulnerability, resilience, and adaptive capacity. Global Environmental Change, 16(3), 293-303.

Griffith, J. L., Agani, F., Weine, S., Ukshini, S., Pulleyblank-Coffey, E., Ulaj, J., \& Kallaba, M. (2005). A Family-based mental health program of recovery from State terror in Kosova. Behavioral Sciences \& the Law, 23(4), 547-558.

Greeff, A. P., Vansteenwegen, A., \& Ide, M. (2006). Resiliency in families with a member with a psychological disorder. American Journal of Family Therapy, 34(4), 285-300.

Henry, K. L., \& Muthén, B. (2010). Multilevel latent class analysis: An application of adolescent smoking typologies with individual and contextual predictors. Structural Equation Modeling: A Multidisciplinary Journal, 17(2), 193-215.

Huang, Y. Q. (2018). Changing intergenerational contracts: Gender, cohorts and elder care in central rural China, 2005-2013. Asian Population Studies, 14(1), 5-21.

Isaacs, S. A., Roman, N. V., Savahl, S., \& Sui, X. C. (2018). Adapting and validating the family resilience assessment scale in an afrikaans rural community in South Africa. Community Mental Health Journal, 54(1), 73-83.

Jiang, C. H., Ma, J. D., Zhang, X., \& Luo, W. J. (2012). Measuring financial protection for health in families with chronic conditions in rural China. BMC Public Health, 12, 988-914.

Kaya, M., \& Arici, N. (2012). Turkish version of shortened Family Resiliency Scale (FRAS): The study of validity and reliability. Procedia: Social and Behavioral Sciences, 55: 512-520.

Lee, Y. H., Cheng, C. Y., \& Lin, S. S. J. (2014). A latent profile analysis of self-control and self-esteem and the grouping effect on adolescent quality of life across two consecutive years. Social Indicators Research, 117(2), 523-539.

Li, C., Li, S. Z., Feldman, M. W., Daily, G. C., \& Li, J. (2012). Does out-migration reshape rural households' livelihood capitals in the source communities? Recent evidence from western China. Asian and Pacific Migration Journal, 21(1), 1-30. 
Li, Y. L., Zhao, Y., Zhang, J., Lou, F. L., \& Cao, F. L. (2016). Psychometric properties of the shortened Chinese version of the family resilience assessment scale. Journal of Child and Family Studies, 25(9), 2710-2717.

Liu, Y. H., \& Xu, Y. (2016). A geographic identification of multidimensional poverty in rural china under the framework of sustainable livelihoods analysis. Applied Geography, 73, 62-76.

Mancini, J. A., Oneal, C. W., Martin, J. A., \& Bowen, G. L. (2018). Community social organization and military families: theoretical perspectives on transitions, contexts, and resilience. Journal of Family Theory \& Review, 10(3), 550-565.

McCubbin, H. I., \& Patterson, J. M. (1983). The family stress process: The double ABCX model of family adjustment and adaptation. Marriage and Family Review, 6(1-2), 7-37.

Muthén, B. O., \& Asparouhov, T. (2009). Multilevel regression mixture analysis. Journal of the Royal Statistical Society, Series A, 172(3), 639-657.

Muthén, L. K. \& Muthén, B.O. (8th ed.). (2017). Mplus user's guide. Los Angeles: Muthén \& Muthén, OECD.

Noor, N. M., Gandhi, A. D., Ishak, I., \& Wok, S. (2014). Development of indicators for family well-Being in Malaysia. Social Indicators Research, 115(1), 279-318.

Nylund, K., Bellmore, A., Nishina, A., \& Graham, S. (2007). Subtypes, severity, and structural stability of peer victimization: What does latent class analysis say? Child Development, 78(6), 1706-1722.

Pandey, R., KumarJha, S., Alatalo, J. M., Archie, K. M., \& Gupta, A. K. (2017). Sustainable livelihood framework-based indicators for assessing climate change vulnerability and adaptation for himalayan communities. Ecological Indicators, 79, 338-346.

Patterson, J. M. (2002). Integrating family resilience and family stress theory. Journal of Marriage and Family, 64(2), 349-360.

Raffaelli, M., Tran, S. P., Wiley, A. R., Galarza-Heras, M., \& Lazarevic, V. (2012). Risk and resilience in rural communities: The Experiences of immigrant Latina mothers. Family Relations, 61(4), 559-570.

Rocchi, S., Ghidelli, C., Burro, R., Vitacca, M., Scalvini, S., Vedova, A. M. D., \& Bertolotti, G. (2017). The walsh family resilience questionnaire: The Italian version. Neuropsychiatric Disease and Treatment, 13, 2987-2999.

Rus, K., Kilar, V., \& Koren, D. (2018). Resilience assessment of complex urban systems to natural disasters: A new literature review. International Journal of Disaster Risk Reduction, 31, 311-330.

Sixbey, M. T. (2005). Development of the Family Resilience Assessment Scale to Identify Family Resilience Constructs (Doctor's thesis). Gainesville, United State: University of Florida.

Taylor, S. D., \& Distelberg, B. (2016). Predicting behavioral health outcomes among low-income families: testing a socioecological model of family resilience determinants. Journal of Child and Famly Studies, 25(9), 2797-2807.

Ungar, M. (2011). The social ecology of resilience: addressing contextual and cultural ambiguity of a nascent construct. American Journal of Orthopsychiatry, 81(1), 1-17.

Vermunt, J. K. (2008). Latent class and finite mixture models for multilevel data sets. Statistical Methods in Medical Research, 17(1), 33-51.

Vesely, C. K., Letiecq, B. L., \& Goodman, R. D. (2017). Immigrant family resilience in context: Using a community-based approach to build a new conceptual model. Journal of Family Theory \& Review, 9(1), 93-110.

Walsh, F. (2003). Family resilience: A framework for clinical practice. Family Process, 42(1), 1-18.

Walsh, F. (2007). Traumatic loss and major disasters: strengthening family and community resilience. Family Process, 46(2), 207-227.

Walsh, F. (2016). Applying a family resilience framework in training, practice, and research: Mastering the art of the possible. Family Process, 55(4), 616-632.

Wang, C. C., Wang, Y., Fang, H. Y., Gao, B., Weng, Z. D., \& Tian, Y. (2020). Determinants of rural poverty in remote mountains of southeast China from the household perspective. Social Indicators Research, $148(3), 1-18$.

Wang, J. J., \& Tian, Q. (2014). Consumer vulnerability and marketplace exclusion: A case of rural migrants and financial services in China. Journal of Macro marketing, 34(1), 45-56.

Wen, Y., \& Hanley, J. (2015). Rural to urban migration, family resilience, and policy framework for social support in China. Asian Social Work and Policy Review, 9(1), 18-28.

Windl, G., Bennett, K. M., \& Noyes, J. (2011). A methodological review of resilience measurement scales. Health and Quality of Life Outcomes, 9, 8-27.

Wu, C., Fu, Q., Gu, J. X., \& Shi, Z. L. (2018). Does migration pay off? returnees, family background, and self-employment in rural China. China Review, 18(1), 59-78.

Yang, J., \& Mukhopadhaya, P. (2017). Disparities in the level of poverty in China: Evidence from China family panel studies 2010. Social Indicators Research, 132(1), 411-450. 
Yang, Y., \& Xu., Y. . (2019). Land endowment and education investment behavior of rural households: a field survey based on 887 administrative villages in 31 provinces of China. The Journal of Chinese Sociology, 6, 3-22.

Zhang, H. F. (2016). Household vulnerability and economic status during disaster recovery and its determinants: A case study after the wenchuan earthquake. Natural Hazards, 83(3), 1505-1526.

Zhang, L. J., Tang, Y., \& Liu, B. H. (2015). Changes in agricultural system as farmers adapt to economicsocial and climatic changes in the min upriver rural areas in western Sichuan, southwestern China. Journal of Mountain Science, 12(3), 747-758.

Publisher's Note Springer Nature remains neutral with regard to jurisdictional claims in published maps and institutional affiliations. 